\title{
The ATLS course, a survey of 228 ATLS providers
}

\author{
D W G Kennedy, D Gentleman
}

\begin{abstract}
Summary
Advanced Trauma Life Support (ATLS) courses teach a system for the initial assessment and management of trauma patients that aims to optimise initial care and reduce mortality and morbidity, and have been adopted worldwide. This questionnaire survey characterised those who took up this particular educational resource in Scotland during a four year period after its introduction, and analysed how they felt it had affected their clinical competence. Irrespective of their previous level of training and experience, nearly all surgeons and anaesthetists who took this course felt that it had improved their clinical skills and other professional attributes. The significance of these results is discussed in the context of postgraduate surgical and anaesthetic training in Scotland.
\end{abstract}

(Emerg Med f 2001;18:55-58)

Keywords: ATLS course

The first Advanced Trauma Life Support (ATLS) course was held in 1978 in Nebraska. It was adopted and patented by the American College of Surgeons Committee on Trauma (ACSCOT) the following year, and since then has spread from the USA to become an integral part of surgical education in many countries. ${ }^{1}$ ATLS courses have a uniform pattern:16 doctors from a mix of specialties relevant to trauma care are taught by a faculty of 6 to 10 trained instructors, who apply adult education theory to inculcate a systematised and collaborative approach to trauma care. ${ }^{23}$ Strong emphasis on the individual tuition of candidates allows formative as well as summative assessment of individual candidates. The course depends upon the enthusiasm of candidates as well as instructors, and teaching is carried out in a style that is consciously positive. The relevance and validity of the course content is strengthened by the clinical credentials of those who teach it, and by four yearly updates conducted by ACSCOT. The 1997 revision has increased contact teaching, and raised the number of instructors needed for a faculty to underline the crucial importance of a high ratio of teachers/mentors/evaluators to learners.

Currently 13000 doctors are trained in ATLS annually in 750 courses worldwide, implying a large investment of time and money. In the United Kingdom the ATLS programme has expanded rapidly since its introduction in 1988, and the Royal Colleges of Surgeons, the Royal College of Anaesthetists and the Faculty of Accident and Emergency Medicine now strongly recommend that all candidates sitting their postgraduate examinations should have completed an ATLS course.

The aim of this survey was to ascertain who in Scotland completed an ATLS course during the four years after its introduction, what those doctors thought of it, and what practical difficulties have emerged.

\section{Methods}

A questionnaire was sent in 1995 to all participants at the 21 ATLS courses held in Scotland between 1991 and 1994 inclusive who could be located. The questionnaire had previously been piloted on a sample group of 32 doctors who attended two courses in Dundee. The returned forms were analysed to ascertain the demographic profile of the candidates.

The respondents were asked what they thought were the most useful parts of the course, using a simple analogue scale (1, no use; 2 , little use; 3 , average; 4 , useful; 5 , very useful). A similar scale was used when they were asked how relevant the course was to their own work. They were also asked how well prepared they felt they were before their course. They were asked, in the context of managing a patient with serious trauma, to grade their knowledge, ability and confidence before and after the course. Questions were also asked about changes in their clinical practice and teaching skills, and their views on a proposal to make an ATLS certificate compulsory for the FRCS and FRCA examinations. They were also asked about difficulties in obtaining study leave and funding to attend the course.

\section{Results}

In all 336 questionnaires were sent out and 228 (68\%) were returned. Table 1 shows the centres that the respondents attended.

Table 2 shows the specialties of the respondents at the time of doing the course. The "other surgery" group includes doctors from surgical rotations, maxillofacial, cardiothoracic, paediatric, vascular, plastic, endoscopic, ENT and neurosurgery. The "others" group includes doctors in general medicine, occupational medicine and sports medicine.

Table 3 shows the grade of respondent at time of reply.

Table 1

$\begin{array}{lc}\text { Glasgow } & 9 \\ \text { Edinburgh } & 5 \\ \text { Dundee } & 4 \\ \text { Irvine } & 1 \\ \text { Elgin } & 1 \\ \text { Aberdeen } & 8 \\ \text { Total } & 2\end{array}$

Mr Kennedy, Specialist Registrar

(DWGKENNEDY@aol.com)

Accepted 27 April 2000 
Table 2

\begin{tabular}{ll}
\hline Anaesthetics & 61 \\
General surgery & 46 \\
Orthopaedics & 43 \\
Accident/emergency & 26 \\
General practice & 21 \\
Other surgery & 22 \\
Others & 9
\end{tabular}

\section{Table 3}

Consultant/Senior Lecturer
Assoc Specialist/Staff Grade
GP Principal
Senior Registrar/Lecturer
Career Registrar
SHO 3
SHO
Research Fellow
GP Trainee
Clinical Assistant
Sports Physician

Sports Physician

Table 4

\begin{tabular}{ll}
\hline Well prepared & $74(32.5 \%)$ \\
Adequately prepared & $127(55.7 \%)$ \\
Poorly prepared & $23(10.1 \%)$ \\
No answer & $4(1.7 \%)$ \\
\hline
\end{tabular}

Each doctor was asked to grade the usefulness of each component of the ATLS course to themselves, using a scale from 1 to 5 . The lecture that was perceived to be the most useful was chapter 1 (Initial Assessment and Management), which scored an average of 4.43 on the 1 to 5 scale. The lecture perceived to be least useful was chapter 8 (Extremity Trauma), which none the less scored 3.59. General practitioners gave the highest scores per chapter (average of 4.32) and orthopaedic surgeons the lowest (average of 3.50). The lowest score from any group was 2.82 , by the anaesthetists for the chapter on Airway and Ventilatory Management.

Respondents were also asked to grade how useful they felt the skills stations were, using the same scale. The highest scoring skills stations were for the moulage practice (4.57) and the moulage test (4.54). The lowest scoring were Vascular Access and Shock Management (3.83) and Head and Neck Trauma Assessment (3.95).

Table 4 shows how prepared the respondents felt they had been for their course, in terms of reading the manual to learn the core material.

Each respondent was asked whether they felt the course had improved their clinical practice, and if they felt it had, were asked to say if this improvement was small, medium or large (table 5). Improvement was reported by 221 respondents $(96.6 \%)$, who were then analysed by seniority and degree of reported improvement (fig 1). The other seven (3\%) reported no improvement: four consultants, two staff grade doctors, and one who was repeating the course to try to achieve instructor status. One respondent stated this question was not applicable because of her teaching, non-clinical role.

Figure 1 shows that most of the "small improvement" group were consultants, most of
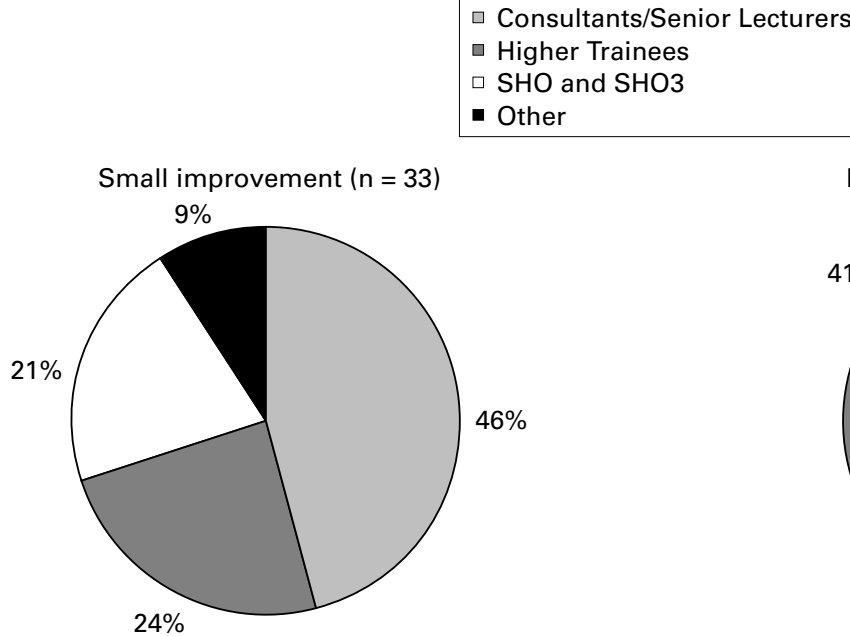

Medium improvement $(n=131)$

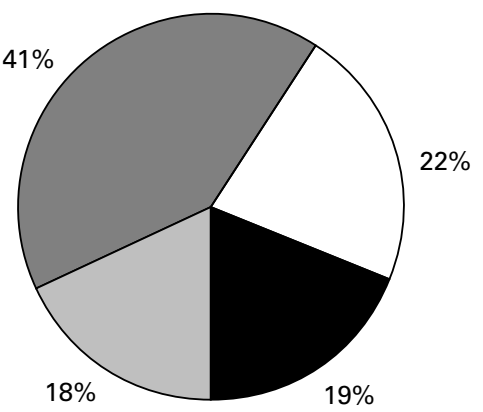

Large improvement $(n=56)$

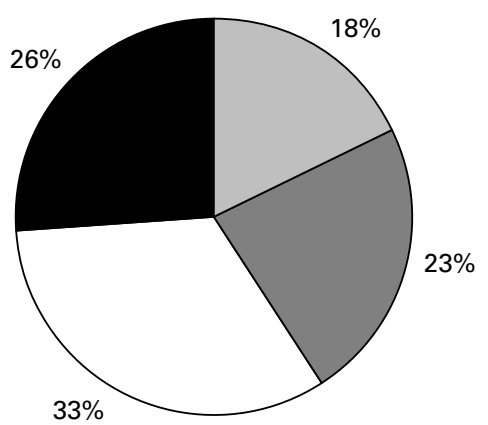

Figure 1 The amount of improvement in clinical practice, subdivided by grade. 
Table 5 Did the ATLS course improve your clinical practice?

\begin{tabular}{lll}
\hline Yes & 220 & $96.6 \%$ \\
No & 7 & $3 \%$ \\
Not applicable & 1 & $0.4 \%$ \\
If yes, by how much? & 33 & $15 \%$ \\
Small improvement & 131 & $60 \%$ \\
Medium improvement & 56 & $25 \%$ \\
Large improvement & & \\
\hline
\end{tabular}

Table 6

Intensity reduced by:

Cutting back lectures

Cutting back skills stations

Increasing time available

Not specified

\begin{tabular}{l}
5 \\
0 \\
32 \\
1 \\
\hline
\end{tabular}

the "medium improvement" group were experienced trainees, and most of the "large improvement" group were inexperienced trainees in the SHO grade. However, all grades were represented in each group, implying that the degree of improvement was not merely influenced by the grade, and therefore the experience, of the candidates. In response to a question on whether the ATLS course had improved their own teaching of trauma management, 213 respondents $(93.4 \%)$ replied that it had, and only $11(4.8 \%)$ reported no improvement.

Thirty eight respondents (16.7\%) thought the course was too intensive, and table 6 shows views on three possible options of how to reduce this.

Doctors were asked to grade their own ability, confidence and level of core knowledge before and after their course, using a zero to nine Likert scale. Mean results for each group are shown in table 7 .

Only one respondent $(0.4 \%)$ would not recommend the course to doctors junior to him, while $224(98.2 \%)$ would. Three respondents offered no opinion regarding advising junior doctors to complete the course. In addition, $201(88.2 \%)$ would encourage doctors senior to them to take the course, while only eight $(3.5 \%)$ would not. Nineteen respondents offered no opinion to this question.

Table 8 shows whether respondents intended to attend a refresher course in four years time.

In response to a question about funding for the course, 19 doctors $(8.3 \%)$ reported prob- lems with study leave. Some $78.5 \%$ of the candidates had their course paid for by the Study Leave Budget (administered by the postgraduate dean), $8.8 \%$ paid themselves, $8.3 \%$ were paid for by their department or practice, and the remaining $4.4 \%$ from other sources.

Table 9 shows views on whether it should be compulsory for surgeons, anaesthetists, and accident and emergency doctors to do a half day refresher course every four years.

Tables 10 and 11 respectively show views on whether ATLS certification should be compulsory before sitting the FRCS and FRCA examinations.

\section{Discussion}

This survey analysed the returned questionnaires of 228 out of 336 which were sent out. It is therefore a survey of a large cohort of doctors holding ATLS certificates and provides a useful snapshot of their views of the course. We have shown that $97 \%$ of the whole group felt that their clinical practice had been improved by attending the course, and that $25 \%$ stated that this was a large improvement. This supports the value of the ATLS course as an educational experience that underpins high quality trauma care. Previous research has shown that levels of core knowledge increase after an ATLS course, ${ }^{45}$ and a similar military course. ${ }^{6}$ This survey confirms that clinical confidence, perception of self, core knowledge and skills, and perceived ability to manage a seriously injured person and to teach others to do so, all increased after attending an ATLS course. These perceived improvements occurred across the board in all grades and specialties, reflected in the finding that $98 \%$ of those surveyed would encourage doctors junior to them to take the course and $88 \%$ would encourage doctors senior to them to attend.

All components of the course received ratings of at least average to useful, except for the predictable rating by anaesthetists of less than average usefulness for Airway and Ventilatory Management. The skills stations were graded very highly, on the 1 to 5 scale used, where 1 represented no use and 5 represented very useful, the skills stations average score was 4.2 , which reflects an overall high opinion of the usefulness of the skills stations. It seems that small group practical skills teaching is

Table 7

\begin{tabular}{|c|c|c|c|c|c|c|c|c|}
\hline & Total & Ortho & Gen Surg & Anaes & $G P$ & $A / E$ & Other Surg & Other \\
\hline \multicolumn{9}{|c|}{ What level were your knowledge and skills } \\
\hline Before your course & 5.32 & 5.35 & 6.02 & 5.9 & 4.86 & 5.65 & 5.80 & 3.66 \\
\hline After your course & 7.80 & 7.72 & 8.04 & 8.04 & 7.71 & 7.88 & 7.90 & 7.33 \\
\hline \multicolumn{9}{|c|}{ How confident were you in the management of a seriously injured patient } \\
\hline Before your course & 5.20 & 5.72 & 5.67 & 5.67 & 4.62 & 5.70 & 5.45 & 3.56 \\
\hline After your course & 7.79 & 8.09 & 8.07 & 8.05 & 7.38 & 7.85 & 7.9 & 7.22 \\
\hline \multicolumn{9}{|c|}{ How capable were you in the management of a seriously injured person } \\
\hline Before your course & 5.24 & 5.65 & 5.96 & 5.79 & 4.86 & 5.6 & 5.5 & 3.33 \\
\hline After your course & 7.76 & 8.00 & 7.91 & 8.08 & 7.43 & 7.77 & 8.04 & 7.11 \\
\hline
\end{tabular}

Table 8

\begin{tabular}{lllllllll}
\hline & Total & Ortho & Gen Surg & Anaes & GP & A/E & Other Surg & Other \\
\hline Yes & 202 & 35 & 41 & 52 & 21 & 23 & 21 & 9 \\
No & 21 & 7 & 4 & 6 & 0 & 3 & 1 & 0 \\
No answer & 5 & 1 & 1 & 3 & 0 & 0 & 0 & 0 \\
\hline
\end{tabular}


Table 9 Should half day refresher courses be compulsory?

\begin{tabular}{llllll}
\hline & Total & All surgeons & Anaes & A/E & All others \\
\hline Yes & 186 & 89 & 47 & 23 & 27 \\
No & 34 & 21 & 10 & 3 & 0 \\
No answer & 8 & 1 & 4 & 0 & 3 \\
\hline
\end{tabular}

Table 10 Should ATLS be compulsory for FRCS?

\begin{tabular}{lll}
\hline & All surgeons & All others \\
\hline Yes & 88 & 87 \\
No & 22 & 21 \\
No answer & 1 & 9 \\
\hline
\end{tabular}

Table 11 Should ATLS be compulsory for FRCA?

\begin{tabular}{lll}
\hline & All anaesthetists & All others \\
\hline Yes & 42 & 107 \\
No & 16 & 35 \\
No answer & 3 & 25 \\
\hline
\end{tabular}

popular. This is reflected in the increased emphasis on this aspect of the course in the 1997 revision.

A large number of the respondents intend to take a refresher course on the expiry of their certificate after four years. Course organisers should be aware of this high level of intent, though whether this will translate into action remains to be seen. Most surgeons $(79 \%)$ and anaesthetists (69\%) think that ATLS Provider status should be compulsory for the FRCS or FRCA examinations respectively. It should be noted, however, that $20 \%$ of the surgeons and $26 \%$ of the anaesthetists did not feel that ATLS should be compulsory for their colleges' examinations. It should be remembered that all of the respondents in this survey have already completed a course and therefore have no vested interest in whether it becomes compulsory. The provision of enough courses to meet such a requirement would raise considerable practical difficulties in all parts of the UK at the present time, mainly because of the shortage of the instructors needed for such an expansion of the programme.

Any educational experience is capable of improvement, and $17 \%$ of the respondents felt that the course was too intensive, although most of them wanted more time and not less course. The 1997 revision has extended the teaching time on each course.
Funding problems were reported by $16 \%$, and study leave problems by $8 \%$. An impression was made during analysis that these problems were most often seen in early courses, and as ATLS became more widely established in Scotland the problems have lessened. This paper, demonstrating the value of the ATLS course in educating doctors involved in trauma care, may help to reduce these problems further. This study shows the breakdown by grade and specialty of those doctors who attended an ATLS course until 1994, and what they thought of it. There has been a change in the types of doctors attending the course, in that there are now many more junior grades attending and it may well be of value to carry out a further study to how much change there has been (W Morrison, personal communication).

In conclusion, this study has shown that the ATLS course is perceived in Scotland as an extremely useful course by doctors in all specialties involved in trauma management, and across all grades of staff. It should therefore be encouraged, but it is less certain that it should be made compulsory. Practical problems like time and money need to be tackled.

\section{Contributors}

Douglas Kennedy initiated this research under the guidance of Douglas Gentleman who advised on the scope and aims of the project. Mr Gentleman advised on the design of the project. $\mathrm{Mr}$ Gentleman advised on the design of the
questionnaire and the questions asked. Douglas Kennedy questionnaire and the questions asked. Douglas Kennedy
distributed the questionnaires, received the replies and collated the data. The text was written jointly by both authors.

Funding: none.

Conflicts of interest: none.

1 Collicut PE. Advanced trauma life support (ATLS). Past, present, future. 16th Stone Lecture.American Trauma Society. J Trauma.1992;33:749-53.

2 American College of Surgeons. ATLS course manual. Chicago: ACS, 1997.

3 Anderson ID, Anderson IWR, Clifford P, et al. Advanced trauma life support in the UK: 8 Years on. Br f Hosp Med 1997;56:272-3.

4 Gautam V, Heyworth J. A method to measure the value of formal training in trauma management: comparison between ATLS and induction courses. Injury 1995;26:2535.

5 Girdley FM, Birnbaum ML, Cohen DJ, et al. Advanced trauma life support: assessment of cognitive achievement. Mil Med 1993;158:623-7.

6 Kluger Y, Rivkind A, Donchin Y, et al. A novel approach to military combat trauma education. I Trauma 1991;31: $564-9$. 